\title{
Modified Arachnoid Plasty Reduces Chronic Subdural Hematoma after Unruptured Aneurysm Clipping : Technical Note
}

\author{
Won Jae Lee, M.D., ${ }^{1}$ Taek Min Nam, M.D., ${ }^{1}$ Kyung-Il Jo, M.D., ${ }^{2}$ Je Young Yeon, M.D., ${ }^{1}$ Seung-Chyul Hong, M.D., Ph.D., \\ Jong-Soo Kim, M.D., Ph.D.' \\ Department of Neurosurgery, Samsung Medical Center, Sungkyunkwan University School of Medicine, Seoul, Korea \\ Department of Neurosurgery, ${ }^{2}$ Hana General Hospital, Cheongju, Korea
}

Objective : Chronic subdural hematoma (CSDH) is a rare complication of unruptured intracranial aneurysm (UIA) clipping surgery. To prevent postoperative CSDH by reducing subdural fluid collection, we applied the modified arachnoid plasty (MAP) during the UIA clipping surgery to seal the dissected arachnoid plane.

Methods : This retrospective study included 286 patients enrolled from July 2012 to May 2015. We performed arachnoid plasty in all patients, with MAP used after June 17, 2014. Patients were divided into two groups (non-MAP vs. MAP), and by using uni- and multivariate analyses, baseline characteristics, and relationships with postoperative CSDH between the two groups were analyzed. The degree of preoperative brain atrophy was estimated using the bicaudate ratio (BCR) index.

Results : Ten patients (3.5\%) among 286 patients had postoperative CSDH after clipping. Nine (3.1\%) were in the non-MAP group, and one (0.9\%) was in the MAP group. The higher BCR index showed statistical significance with occurrence of postoperative CSDH in both uni- ( $p=0.018)$ and multivariate ( $p=0.012$; odds ratio [OR], 8.547; 95\% confidence interval $[\mathrm{Cl}], 1.616-45.455)$ analyses. MAP was associated with a lower risk of postoperative $\mathrm{CSDH}$ ( $p=0.022 ; \mathrm{OR}, 0.068 ; 95 \% \mathrm{Cl}, 0.007-0.683$ ).

Conclusion : This study shows that the degree of preoperative brain atrophy is associated with an increased occurrence of CSDH after clipping and that MAP could help reduce the risk of postoperative CSDH after unruptured aneurysm clipping via a lateral supraorbital approach.

Key Words : Unruptured aneurysm · Arachnoid plasty · Subdural fluid collection · Chronic subdural hematoma.

\section{INTRODUCTION}

Chronic subdural hematoma (CSDH) is a rare complication of unruptured intracranial aneurysm (UIA) clipping surgery ${ }^{5}$. A previous study suggested that an increase of subdural fluid collection (SDFC) during the first postoperative week may be a risk factor of CSDH after clipping surgery of unruptured aneurysms ${ }^{11)}$. Mino et al..$^{9)}$ reported that arachnoid plasty using fibrin glue prevented subdural fluid collection after ruptured aneurysm clipping surgery. It is plausible that arachnoid

- Received : September 5, 2017 •Revised : October 24, 2017 •Accepted : November 13, 2017

- Address for reprints : Jong-Soo Kim, M.D., Ph.D.

Department of Neurosurgery, Samsung Medical Center, Sungkyunkwan University School of Medicine, 81 Irwon-ro, Gangnam-gu, Seoul 06351, Korea Tel : +82-2-3410-3499, Fax : +82-2-3410-0048, E-mail : jsns.kim@samsung.com

This is an Open Access article distributed under the terms of the Creative Commons Attribution Non-Commercial License (http://creativecommons.org/licenses/by-nc/4.0) which permits unrestricted non-commercial use, distribution, and reproduction in any medium, provided the original work is properly cited. 
plasty may prevent postoperative CSDH by reducing postoperative SDFC, but the techniques and role of arachnoid plasty in unruptured aneurysm clipping have not been fully validat$\mathrm{ed}^{8)}$. The objectives of this study were to introduce the modified arachnoid plasty (MAP) technique during UIA clipping surgery and to evaluate the efficacy of MAP.

\section{MATERIALS AND METHODS}

We retrospectively reviewed the medical records of 379 consecutive patients who underwent clipping surgery for UIA from July 2012 to May 2015 by one senior neurosurgeon at a single institute. We included patients with small to large aneurysms (size $<2.5 \mathrm{~cm}$ ) on anterior circulation, and we only used the lateral supraorbital (LSO) approach as previously described $^{3)}$. We excluded giant or complex aneurysms requiring complicated surgical techniques in addition to clipping $(n=5)$, a bilateral approach in single session $(n=21)$, distal anterior cerebral artery (ACA) aneurysms, and posterior circulation aneurysms $(n=67)$ approached by other than a LSO approach. Among the 379 UIA patients that underwent clipping surgery, a total of 286 patients met the inclusion criteria. We analyzed patient data including age, sex, size and location of aneurysm, preoperative brain atrophy, postoperative SDFC, and usage of antithrombotics ${ }^{13)}$.

Aneurysm sizes were divided into three groups based on maximal diameter in 3-dimensional digital subtracted angiography : tiny $(<5 \mathrm{~mm})$, small $(5-10 \mathrm{~mm})$, and large (10-25 $\mathrm{mm})$. The location of aneurysm was categorized as ACA, middle cerebral artery (MCA), and internal carotid artery (ICA) according to the parent artery. ACA included the anterior communicating artery and proximal A2 and A1 aneurysms. ICA included aneurysms from the paraclinoid segment of ICA to ICA bifurcation. MCA aneurysms were defined as aneurysms on the MCA after the proximal M1 segment.

The degree of preoperative brain atrophy was estimated using the bicaudate ratio (BCR), which is defined as the ratio of the width of ventricles between the caudate nuclei and the internal diameter of the skull at the same level; BCR indices greater than 0.11 were defined as preoperative brain atrophy ${ }^{2}$. BCRs were measured on brain computed tomography (CT) scans that were taken within 6 months prior to surgery. In patients without CT scans, we used magnetic resonance T2weighted imaging scans that were obtained within the same time period.

\section{Techniques}

All patients in this study received arachnoid plasty or MAP during clipping surgery. Before June 2014, the arachnoid plasty technique applied fibrillar (Surgicel Fibrillar, Ethicon Inc., Somerville, NJ, USA) and fibrin glue at the dissected arachnoid surface at the end of surgery as described in a previous study $^{8)}$. The MAP technique was used after June 17, 2014. After clipping the aneurysm, we put a 5 Fr feeding tube into the dissected arachnoid plane through the opened Sylvian fissure and fill the opened space with multiple small pieces of absorbable hemostatic gelatin sponges (SPONGOSTAN ${ }^{\mathrm{TM}}$ Standard, Ethicon Inc.) (Fig. 1A, B). In order to reinforce the sealing of the Sylvian fissure, fibrin glue was applied over the gelatin sponges. We infused normal saline into the subarachnoid space through the feeding tube until the brain, which had sunk due to cerebrospinal fluid (CSF) leakage, was refloated (Fig. 1C). After removing the feeding tube, the entrance hole of the tube was covered with a small piece of gelatin sponge (Fig. 1D). Lastly, the dura mater was closed in a water-tight fashion. According to the method of arachnoid plasty, patients were divided into two groups (non-MAP or MAP). The
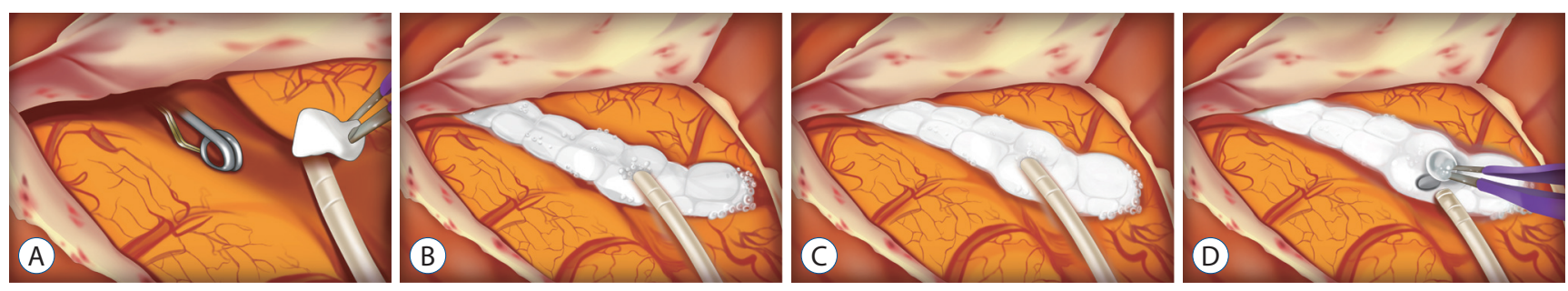

Fig. 1. A : Illustration shows a feeding tube put into the opened Sylvian fissure after clipping. B : The opened arachnoid plane is covered with multiple absorbable hemostatic gelatin sponges. C : After the reinforcement of gelatin sponges covered surface using fibrin glue, we infuse normal saline through the feeding tube. $\mathrm{D}$ : The sunken brain was refloated, and the entrance hole of the tube was plugged with a gelatin sponge. 
opened subarachnoid space after aneurysm clipping and an operative view of gelatin sponge-covered opened Sylvian fissure are shown in Fig. 2, respectively.

Postoperative SDFC was defined as the maximal distance between the inner table of the skull and the cerebral cortex. Before 2014, we checked the brain non-contrast CT on postoperative day 1 and intracranial CT angiography (CTA) on postoperative day 3 or 4 . Patients were typically discharged 5 to 7 days after surgery. After January 2014, intracranial CTAs were performed on postoperative day 1, within 24 hours after clipping, and patients were typically discharged on postoperative day 2 or 3 . We measured maximal vertical depth of the SDFC on the ipsilateral side of the clipped aneurysm on brain non-contrast CT images performed on postoperative day 1 and compared the patients who had SDFC of less than $10 \mathrm{~mm}$ to those with SDFC greater than $10 \mathrm{~mm}$.

Postoperative CSDH was defined as symptomatic CSDH that required surgical drainage. Clinical follow-ups were routinely performed 1 and 6 months after clipping. Scheduled radiologic follow-ups using intracranial CTA were performed after 1 or 2 years in all patients included in this study; however, patients only received imaging studies if necessary. We analyzed baseline characteristics between the two groups (nonMAP vs. MAP) as well as the factors associated with postoperative $\mathrm{CSDH}$.

\section{Statistical analyses}

Mean and frequency comparisons were performed using
Student's t-tests, chi-square tests, Mann-Whitney U tests, or Fisher's exact tests as appropriate. Univariate statistical analyses (chi-square tests or Fisher's exact tests) were performed to assess associations between variables and $\mathrm{CSDH}$, the categorical variable. Variables with $p<0.15$ in univariate analyses were selected for multivariate models using multiple logistic regression analyses. Differences were considered significant at $p<0.05$. Statistical analyses were performed using SPSS version 22.0 software (SPSS Inc., Chicago, IL, USA).

\section{RESULTS}

In total, 286 patients were included in this study; 180 were in the non-MAP group, and 106 were in the MAP group. The mean ages in each of the two groups were 57.03 \pm 9.36 and $57.16 \pm 10.29$, respectively. Baseline characteristics of the two groups are summarized in Table 1. Age, gender, size and location of aneurysms, postoperative SDFC, and the use of antithrombotics showed no statistically significant differences between the two groups, but the MAP group had a higher proportion of patients with preoperative brain atrophy than the non-MAP group (55/180 vs. $63 / 106, p<0.001)$. There were no complications associated with MAP including infection, allergic reaction ${ }^{6}$ or technical failure. Also, there was no infarction or venous congestion due to venous compromise.

Ten patients (3.5\%) of the 286 participants received burr hole trephination due to postoperative $\mathrm{CSDH}$ after clipping.
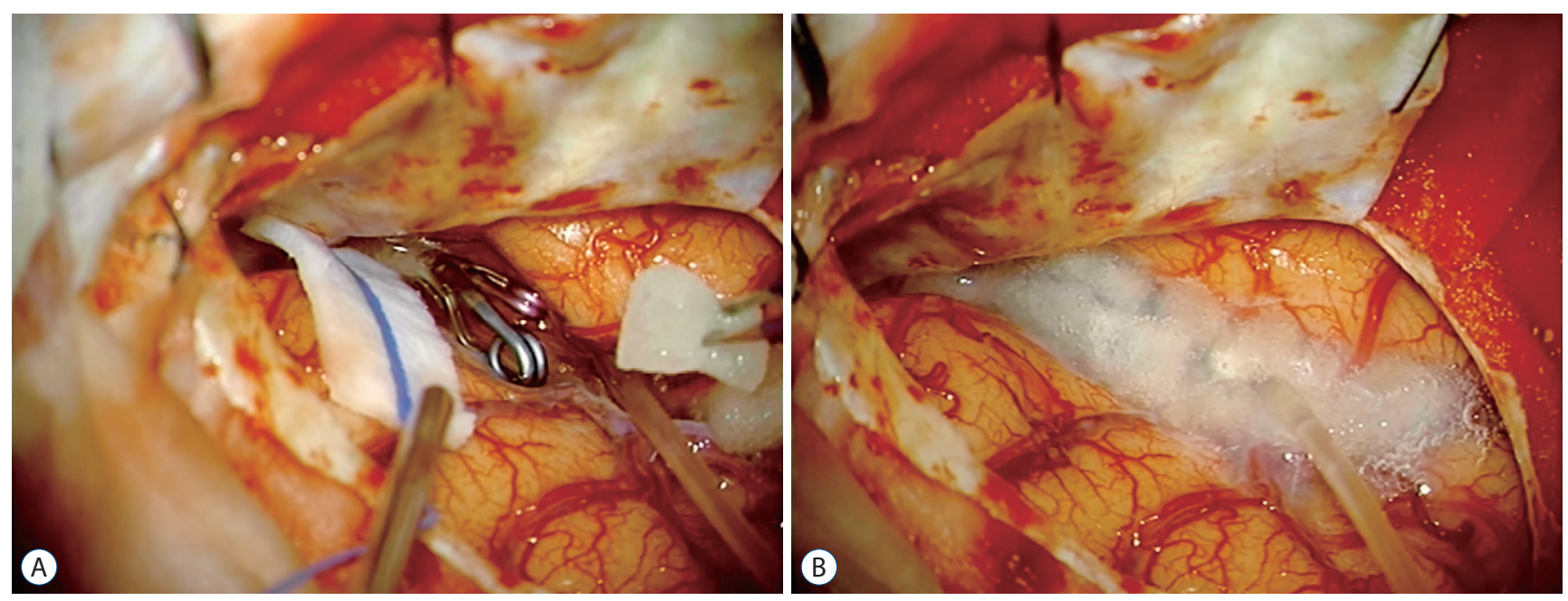

Fig. 2. A : Microscopic view showing surgically dissected arachnoid membrane and opened Sylvian fissure. B : Covered Sylvian fissure using multiple gelatin sponges and fibrin glue. 
Table 1. Baseline characteristics of the study population in the non-MAP vs. MAP groups

\begin{tabular}{|c|c|c|c|}
\hline Characteristic & Non-MAP $(n=180)$ & MAP $(n=106)$ & $p$-value \\
\hline Mean age (years) & $57.03 \pm 9.36$ & $57.16 \pm 10.29$ & 0.921 \\
\hline Male : female & $60(33.3): 120(66.7)$ & $33(31.1): 73(68.9)$ & 0.402 \\
\hline Aneurysm size (mm) & & & 0.370 \\
\hline$<5$ & $105(58.3)$ & $70(66.0)$ & \\
\hline $5-10$ & $70(38.9)$ & $35(33.0)$ & \\
\hline $10-25$ & $5(2.8)$ & $1(0.9)$ & \\
\hline Location of aneurysm & & & 0.559 \\
\hline ACA & $40(22.2)$ & $18(17.0)$ & \\
\hline MCA & $88(48.9)$ & $60(56.6)$ & \\
\hline ICA & $40(22.2)$ & $17(16.0)$ & \\
\hline Multiple & $12(6.7)$ & $11(10.4)$ & \\
\hline$B C R$ index $(>0.11)$ & 55 (30.6) & $63(59.4)$ & $<0.001$ \\
\hline Post-op SDFC (>10 mm) & $5.51 \pm 2.24$ & $5.67 \pm 2.08$ & 0.549 \\
\hline Antithrombotics & $37(20.6)$ & $24(22.6)$ & 0.765 \\
\hline
\end{tabular}

Values are presented as mean \pm standard deviation or number (\%). MAP : modified arachnoid plasty, ACA : anterior cerebral artery, MCA : middle cerebral artery, ICA : internal carotid artery, BCR : bicaudate ratio, op : operative, SDFC : subdural fluid collection

Table 2. Factors associated with the incidence of postoperative CSDH

\begin{tabular}{lccc}
\hline Characteristic & Univariate analysis & Multivariate analysis & OR (95\% Cl) \\
\hline Age $\geq 65$ & 1.000 & & 0.063 \\
Male & 0.015 & 0.080 & $0.258(0.057-1.174)$ \\
ACA location & 0.085 & & \\
Size of aneurysms & 0.342 & $0.928-18.536)$ \\
BCR index $>0.11)$ & 0.018 & 0.690 & $8.547(1.616-45.455)$ \\
Use of Antithrombotics & 0.229 & 0.070 & $0.731(0.157-3.406)$ \\
Post-op SDFC $(>10 \mathrm{~mm})$ & 0.038 & 0.022 & $1.367(0.975-1.918)$ \\
MAP & 0.097 & $0.068(0.007-0.683)$ \\
\hline
\end{tabular}

CSDH : chronic subdural hematoma, OR : odds ratio, $\mathrm{Cl}$ : confidence interval, ACA : anterior cerebral artery, BCR : bicaudate ratio, op : operative, SDFC : subdural fluid collection, MAP : modified arachnoid plasty

No patients with postoperative CSDH had a history of head trauma after clipping. All cases of postoperative CSDH occurred on the ipsilateral side of the clipped aneurysm, and there were no complications associated with surgical drainage of CSDH. Male gender ( $p=0.015)$, higher BCR index ( $p=0.018$ ), and postoperative SDFC greater than $10 \mathrm{~mm}(p=0.038)$ showed statistical significance with the occurrence of postoperative $\mathrm{CSDH}$ in univariate analysis. Among the 10 patients with postoperative $\mathrm{CSDH}$, nine (3.1\%) were in the non-MAP group, and one (0.9\%) was in the MAP group. This difference did not show statistical significance in univariate analysis ( $p=0.097$ ). In multivariate analyses, the higher BCR index was associated with postoperative $\mathrm{CSDH}$ ( $p=0.012$; odds ratio $[\mathrm{OR}], 8.547$; 95\% confidence interval [CI], 1.616-45.455), and MAP was associated with a low risk of postoperative $\mathrm{CSDH}$ ( $p=0.022$; OR, 0.068 ; 95\% CI, 0.007-0.683) (Table 2). Postoperative SDFC was not associated with postoperative CSDH in multivariate analysis.

\section{DISCUSSION}

The reported incidence of CSDH after neurosurgery is $0.8 \%$, and the incidence after clipping surgery is $2.4 \%{ }^{10)}$. Sev- 
eral studies have reported the risk factors of CSDH after aneurysm clipping ${ }^{5,8,11)}$, and the one of these studies reported that increased postoperative SDFC was associated with higher risk of postoperative CSDH in clipping surgery ${ }^{11}$. The opening of subarachnoid cisterns during clipping surgery allows the communication of CSF between the subarachnoid and subdural spaces $^{14,16,17)}$. Postoperative SDFC is the result of CSF leakage from surgically dissected arachnoid membranes ${ }^{9,10)}$. To reduce SDFC by sealing this opened space, we applied arachnoid plasty for all patients. MAP was associated with a low risk of postoperative CSDH in this study, and there was no significant difference of SDFC between the non-MAP and MAP groups. Preoperative brain atrophy can cause enlargement of the subarachnoid space and provide a wide potential space for fluid accumulation ${ }^{12)}$, but our previous data showed that there was no statistically significant difference in SDFC between the higher BCR index group and the lower BCR index group ${ }^{8)}$. These findings imply that the single measurement of SDFC on immediate postoperative images did not reflect the exact amount and changes of SDFC during the follow-up period and that it is not a predicting factor associated with the occurrence of postoperative CSDH. A recent study performed arachnoid plasty using collagen sheets and fibrin glue reported that there was no SDFC on brain CT obtained 1 month after clipping surgery, although there was no immediate measurement of $\mathrm{SDFC}^{1}$. Controlled prospective studies investigating preoperative SDFC and postoperative SDFC changes by serial imaging follow-up might show different result.

There were no standard procedures and definite indications of arachnoid plasty ${ }^{1}$. A recent study suggested that arachnoid plasty had the possibility to prevent CSDH after unruptured aneurysm clipping and the technique of arachnoid plasty was very similar to MAP. But they did not consider the different backgrounds of patients who did or did not receive arachnoid plasty $^{15)}$. We did arachnoid plasty in all patients who underwent clipping surgery and divided them into two groups according to the technical differences in arachnoid plasty (nonMAP and MAP). Several techniques of arachnoid plasty have been introduced to reduce postoperative SDFC-associated complications $^{1,8,16}$. The MAP described in this study does not require difficult surgical techniques or extra time. Also, we did not observe any complications associated with MAP including infection or allergic reaction. Since there were no significant differences of baseline characteristics between the two groups except for the degree of preoperative brain atrophy, our findings suggest that MAP had a preventive effect on occurrence of postoperative CSDH.

The method of non-MAP applied fibrillar (Surgicel Fibrillar, Ethicon Inc.) on the opened Sylvian fissure after filling the dissected CSF cisterns and subarachnoid spaces with normal saline. And then, we covered the fibrillar surface using fibrin glue $^{8}$. In MAP, we packed the Sylvian fissure using multiple small pieces of gelatin sponges. Because these sponges can absorb water and expand, they can compress the frontal and temporal lobe surfaces and provide a tighter seal than the fibrillar compound. Additionally, saline filling after sealing the dissected plane could fill the opened subarachnoid spaces more effectively, which might contribute to reducing SDFC. This was supported by the findings that there was no difference in SDFC between the non-MAP and MAP groups despite the MAP group having a higher proportion of brain atrophy.

Although there was no unexpected event by hemostatic materials in our study, there are concerned about complications, such as chemical meningitis, venous compromise, hydrocephalus, allergic reaction and foreign body granuloma. Granulomatous or allergic reaction to foreign bodies, such as fibrin glue and gelatin sponge, has been described in previous studies $^{4,6,7)}$. These materials have been widely used as a hemostatic agent during neurosurgical procedures. However, foreign body granuloma and allergic reaction caused by resorbable hemostatic agents is an extremely rare condition. It is necessary that the surgeon be aware of these potential events, however, it does not seem to be a concern.

Several limitations should be noted in this study. First, this study was retrospective in nature. Serial follow-up postoperative brain images were lacking despite the fact that changes in postoperative SDFC were known to be an important predictor of postoperative CSDH. Second, this study did not have a randomized control group. Therefore, different characteristics of patients could influence the study outcome even though statistically significant differences were not observed.

\section{CONCLUSION}

In this study, we demonstrated that the degree of preoperative brain atrophy is associated with the occurrence of $\mathrm{CSDH}$ 
after aneurysm clipping and that MAP is associated with lowering the risk of postoperative CSDH. MAP seems to be a simple, safe and effective method to prevent CSDH after UIA clipping. Further prospective controlled studies are needed to confirm these findings.

\section{CONFLICTS OF INTEREST}

No potential conflict of interest relevant to this article was reported.

\section{INFORMED CONSENT}

Informed consent was obtained from all individual participants included in this study.

\section{References}

1. Abe J, Ichinose T, Terakawa $Y$, Tsuyuguchi N, Tsuruno T, Ohata K : Efficacy of arachnoid plasty with collagen sheets and fibrin glue: an in vitro experiment and a case review. Surg Neurol Int 6 : 90, 2015

2. Aylward EH, Schwartz J, Machlin S, Pearlson G : Bicaudate ratio as a measure of caudate volume on MR images. AJNR Am J Neuroradiol 12 : 1217-1222, 1991

3. Cha KC, Hong SC, Kim JS : Comparison between lateral supraorbital approach and pterional approach in the surgical treatment of unruptured intracranial aneurysms. J Korean Neurosurg Soc 51 : 334-337, 2012

4. Hasturk $A E$, Basmaci $M$ : Foreign body granuloma mimicking recurrent intracranial tumor: a very rare clinical entity. Acta Med Iran 51 : 816818,2013

5. Inamasu J, Watabe T, Ganaha T, Yamada Y, Nakae S, Ohmi T, et al. : Clinical characteristics and risk factors of chronic subdural haematoma associated with clipping of unruptured cerebral aneurysms. J Clin Neu- rosci 20 : 1095-1098, 2013

6. Kanazawa R, Sato S, Iwamoto N, Teramoto A : Allergic reaction following arachnoid plasty with a fibrin sealant. Neurol Med Chir (Tokyo) $50: 608-610,2010$

7. Kothbauer KF, Jallo GI, Siffert J, Jimenez E, Allen JC, Epstein FJ : Foreign body reaction to hemostatic materials mimicking recurrent brain tumor. report of three cases. J Neurosurg 95 : 503-506, 2001

8. Lee WJ, Jo KI, Yeon JY, Hong SC, Kim JS : Incidence and risk factors of chronic subdural hematoma after surgical clipping for unruptured anterior circulation aneurysms. J Korean Neurosurg Soc 57 : 271-275, 2015

9. Mino Y, Hirashima Y, Hamada H, Masuoka T, Yamatani K, Takeda S, et al. : Effect of arachnoid plasty using fibrin glue membrane after clipping of ruptured aneurysm on the occurrence of complications and outcome in the elderly patients. Acta Neurochir (Wien) 148 : 627-631; discussion 631, 2006

10. Mori K, Maeda M : Risk factors for the occurrence of chronic subdural haematomas after neurosurgical procedures. Acta Neurochir (Wien) 145 : 533-539; discussion 539-540, 2003

11. Ohno T, lihara K, Takahashi JC, Nakajima N, Satow T, Hishikawa T, et al. : Incidence and risk factors of chronic subdural hematoma after aneurysmal clipping. World Neurosurg 80 : 534-537, 2013

12. Quintana LM : Chronic subdural hematoma after neurosurgical procedures. World Neurosurg 80 : 482-483, 2013

13. Sim YW, Min KS, Lee MS, Kim YG, Kim DH : Recent changes in risk factors of chronic subdural hematoma. J Korean Neurosurg Soc 52 : 234-239, 2012

14. Takahashi Y, Ohkura A, Sugita Y, Sugita S, Miyagi J, Shigemori M : Postoperative chronic subdural hematoma following craniotomy--four case reports. Neurol Med Chir (Tokyo) 35 : 78-81, 1995

15. Yagi $K$, Irie $S$, Inagaki $T$, Ishii $Y$, Saito 0 , Lee $T$, et al. : Intraoperative arachnoid plasty has possibility to prevent chronic subdural hematoma after surgery for unruptured cerebral aneurysms. Neurol Med Chir (Tokyo) 55 : 493-497, 2015

16. Yoshimoto $T$, Houkin $K$, Ishikawa $T$, Abe H : Arachnoid membrane closure. prevention of postoperative cerebrospinal fluid leakage. Surg Neurol 52 : 68-71; discussion 71-72, 1999

17. Yoshimoto Y, Wakai S, Hamano M : External hydrocephalus after aneurysm surgery: paradoxical response to ventricular shunting. J Neurosurg $88:$ 485-489, 1998 